\title{
Time-like proton form factors and heavy lepton production at the PANDA experiment
}

\author{
Alaa Dbeyssi ${ }^{* \dagger}$ \\ IPN-Orsay \\ E-mail: dbeyssi@ipno.in2p3.fr
}

Electromagnetic form factors (FFs) are fundamental quantities which describe the structure and the internal dynamics of hadrons. Assuming one-photon exchange, the production of electronpositron pairs in proton-antiproton annihilation allows to access electromagnetic FFs in the timelike region of the momentum transfer squared. At the future accelerator complex FAIR (Facility for Antiproton and Ion Research), in Darmstadt, the PANDA (antiProton ANnihilation at DArmstadt) collaboration plans to measure proton time-like FFs using an antiproton beam of momentum up to $15 \mathrm{GeV} / \mathrm{c}$ and luminosity $\mathscr{L}=2.10^{32} \mathrm{~cm}^{-2} \mathrm{~s}^{-1}$. In the present work the annihilation of an antiproton beam on a proton target into heavy leptons as muons or taus is considered. Calculating the production of taus or muons, the leptons mass can not be neglected, as it is generally done for electrons. The interest of FFs measurements with heavy leptons is related to the fact that radiative corrections are strongly suppressed. Moreover, polarization observables which are proportional to the lepton mass are enhanced. The differential cross section and polarization observables, when the beam and/or target are polarized, as well as the polarization of the outgoing leptons in $\bar{p}+p$ annihilations are derived at the Born approximation. The effect of the additional terms induced by the lepton mass on the observables is investigated. A numerical application is done using the VDM parametrization of proton FFs. Our results show an enhancement of the polarization observables of these heavy particles, in particular when the transverse polarization of the leptons is considered. The measurement of unstable lepton polarization can be done through the angular distribution of the decay products.

International Winter Meeting on Nuclear Physics,

21-25 January 2013

Bormio, Italy

* Speaker.

†n the behalf of the PANDA collaboration. 


\section{Introduction}

The PANDA (antiProton ANnihilation at Darmstadt) experiment [1] is one of the projects planned at the future Facility for Antiproton and Ion Researches (FAIR) [2], which is under construction next to GSI, Darmstadt. The PANDA physics programme aims to achieve precise studies and quantitative understanding of the non perturbative low energy Quantum Chromodynamics (QCD). QCD is well understood at high energies, where the strong coupling constant is small and perturbation theory can be applied. The broad scientific programme of PANDA covers charmonium spectroscopy, search for gluonic excitations, open charm spectroscopy, hypernuclear physics and electromagnetic processes. The experiment will benefit from the high intensity antiproton beams which will be provided by the high energy storage ring (HESR) of FAIR, in the momentum range from $1.5 \mathrm{GeV} / \mathrm{c}$ to $15 \mathrm{GeV} / \mathrm{c}$. For the PANDA detector, a good particle identification with almost $4 \pi$ acceptance and electromagnetic calorimetry will be combined with high resolution tracking to achieve the proposed physics goals.

The understanding of the complex structure of nucleon is one of the main goals of PANDA experiment. The elastic electron nucleon $(e N)$ scattering, where the interaction occurs through the exchange of a virtual photon, is an effective tool to investigate in space-like (SL) region the nucleon electromagnetic structure, which is parametrized in terms of electric and magnetic form factors (FFs). Electromagnetic FFs are related to the charge distribution and the magnetization current within the nucleon. This relation is straightforward in non-relativistic approximation or in the Breit frame.

The electromagnetic FFs are real functions of the momentum transfer squared in the SL region corresponding to the $e N$ scattering, and become complex in the time-like (TL) region which is accessible in the annihilation channels, $\bar{N}+N \leftrightarrow e^{+}+e^{-}$.

The SL proton FFs have been first measured using the Rosenbluth separation method. The recent availability of high intensity and high polarized electron beams at JLab allowed their precise measurement up to large values of the momentum transfer squared by the GEp collaboration, using the polarization method suggested by Akhiezer and Rekalo in 1968. In the TL region, due to the low luminosity achieved up to now, few data exist on the nucleon FFs and no individual determination of the electric and magnetic FFs has been done. Monte Carlo simulations [3] show that at PANDA, the absolute cross section can be measured up to a value of the momentum transfer squared $q^{2} \sim 28$ $\mathrm{GeV}^{2}$, and the electric and magnetic FFs can be separately determined up to $14 \mathrm{GeV}^{2}$.

The reaction $\bar{p} p \rightarrow e^{+} e^{-}$has been first studied in Ref. [4] in connection with the possibility to extract proton FFs in the annihilation region, assuming one photon exchange. Polarization observables have been derived in the assumption of $m_{e}=0$ in Ref. [5]. Following the formalism of Ref. [6], we extend the calculations of polarization observables for the annihilation reaction $\bar{p}+p \rightarrow e^{-}+e^{+}$to the case of heavy leptons, such as $\mu$ or $\tau$. The main difference is that the mass of the lepton can not be neglected and this induces new terms in the structure of the amplitude. The interest of FFs measurement in $\bar{p}+p$ annihilation into heavier leptons is related to the following facts:

- The individual determination of the electric and magnetic proton form factors in $\bar{p} p \rightarrow \ell^{+} \ell^{-}$ requires a precise measurement of the angular distribution of the final lepton for the Born 
diagram. Radiative corrections (RC) due to the emission of real and virtual photons do affect the measurement of the experimentally observable quantities, in particular of the differential cross section. Based on a model for the proton FFs, one needs to evaluate theoretically the radiative correction due to the final state radiation, namely photon emission from both leptons, as well as the radiation in the initial state at the proton vertex.

Full simulation with PANDA detector showed that the differential cross section can be measured at PANDA with a precision of about 3\%-5\%. Analytical calculations [7] for $\bar{p} p \rightarrow e^{+} e^{-}$showed a total value of RC in the range [20\%-40\%] [8], depending on the energy and the angle of the outgoing electrons. In the case of muon or tau production, RC are essentially suppressed by the mass of the heavy leptons.

- The $\tau$ lepton is an unstable particle with a decay time equal to $290.6 \times 10^{-15} \mathrm{~s}$. The polarization of unstable particles can be measured, in principle, through the angular or energy distribution of their weak decay products; by consequence, the observables related to the lepton polarization may be experimentally measured without polarimeters. At the LEP collider, the ALEPH Collaboration measured the $\tau$ polarization in the center of mass (CM) of electronpositron collision from different decays such as $\tau \rightarrow \pi^{-}+v_{\tau}, \tau \rightarrow a_{1}+v_{\tau}, \tau \rightarrow \rho+v_{\tau}$ $[9,10]$.

- From the relativistic definition of the particle polarization 4-vector, the observables corresponding to the transverse polarization of the lepton contain the factor $m_{\ell} / E\left(m_{\ell}\right.$ is the lepton mass, $E$ is the incident energy). In the $\mathrm{GeV}$ range, this corresponds to a huge suppression in case of electron, whereas, in case of $\tau$ lepton it becomes an enhancement in the $\mathrm{GeV}$ range.

\section{General formalism for (heavy) lepton pair production}

Let us consider the reaction:

$$
\bar{p}\left(p_{1}\right)+p\left(p_{2}\right) \rightarrow \ell^{-}\left(k_{1}\right)+\ell^{+}\left(k_{2}\right)
$$

where $\ell=e, \mu$ or $\tau$ and the four-momenta of the particles are written in parentheses. In the Born approximation $q=k_{1}+k_{2}=p_{1}+p_{2}$ is the four momentum of the exchanged virtual photon.

\subsection{Kinematics}

In the CM system, the lepton pair is emitted back to back and each lepton carries half of the total energy. In the Lab system, the kinematics for a massive lepton, in particular for a $\tau$-lepton, which mass is larger than the proton mass, is essentially different from the case when the lepton mass is neglected. In the case of $e$ or $\mu$, there is no limitation on the angular region of the produced (negative) lepton in the Lab system, and there is a unique relation between the energy and the angle. In the limit of zero lepton mass, this relation can be written as:

$$
E_{1}=\frac{M W}{W-p \cos \theta_{1}}
$$




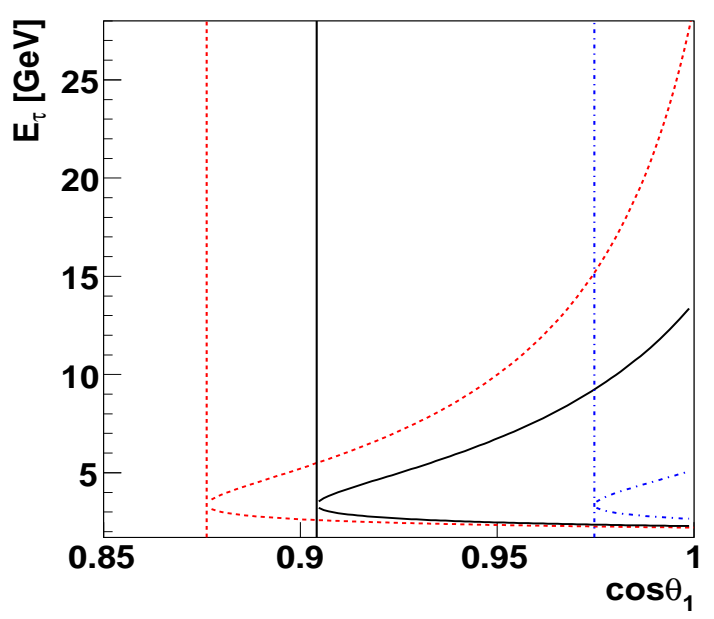

Figure 1: Energy of the $\tau^{-}$lepton as a function of the emission angle for $E=6.85 \mathrm{GeV}$ (blue dot-dashed line), $E=15 \mathrm{GeV}$ (black solid line) and $E=30 \mathrm{GeV}$ (red dashed line), in the Lab system. The limiting angles are shown as vertical lines for the corresponding energy.

When the mass of the lepton exceeds the proton mass, there is a maximum limiting angle for the lepton emission $\theta_{\max }$, which depends on the lepton mass and on the incident energy:

$$
\cos \theta_{\max }=\frac{W \sqrt{m_{\ell}^{2}-M^{2}}}{m_{\ell} p},
$$

and it is illustrated as vertical lines in Fig. 1.

In the Lab system, from the conservation laws of energy and momentum one finds that one angle corresponds to two possible values for the energy of the emitted $\tau^{-}$lepton:

$$
E_{1}^{ \pm}=\frac{M W^{2} \pm \sqrt{p^{2} \cos ^{2} \theta_{1}\left[W^{2}\left(M^{2}-m_{\ell}^{2}\right)+m_{\ell}^{2} p^{2} \cos ^{2} \theta_{1}\right]}}{\left(W^{2}-p^{2} \cos ^{2} \theta_{1}\right)} .
$$

This is illustrated in Fig. 1, for three incident energies $E=6.85 \mathrm{GeV}$, just above the kinematical threshold (blue dash-dotted line), $E=15 \mathrm{GeV}$ (black solid line) and $E=30 \mathrm{GeV}$ (red dashed line), well above threshold.

The correlated lepton has two values for the energy (which satisfy energy conservation) and is emitted at two corresponding angles (Fig. 2).

\section{Matrix element}

The scattering amplitude $\mathscr{M}$ is a relativistic invariant. In a Feynman gauge, $\mathscr{M}$ can be written in terms of the electromagnetic current of the lepton $\left(j_{\mu}\right)$, the nucleon $\left(J_{\mu}\right)$ and the photon propagator as:

$$
\mathscr{M}=-\frac{4 \pi \alpha}{q^{2}} j_{\mu} J_{\mu}=-\frac{4 \pi \alpha}{q^{2}}\left(j_{0} J_{0}-\vec{j} \vec{J}\right)
$$




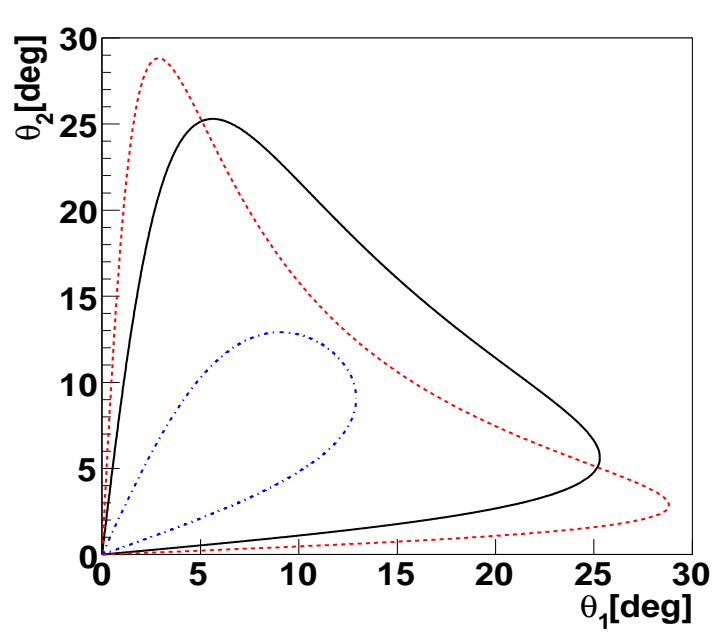

Figure 2: $\tau^{+}$emission angle $\theta_{2}$ as a function of the $\tau^{-}$angle $\theta_{1}$ in the Lab system. Notation as in Fig. 1.

where $\alpha$ is the electromagnetic coupling constant. The leptonic current, assuming no internal structure for the lepton, can be derived from the Lagrangian density of the quantum electrodynamics (QED) as:

$$
j_{\mu}=\bar{u}\left(k_{1}\right) \gamma_{\mu} v\left(k_{2}\right)
$$

where $u\left(k_{1}\right)\left(v\left(k_{2}\right)\right)$ is a four component spinor of free Dirac particle (antiparticle). The structure of the hadronic current is more complicated than the leptonic current due to the complex nature of nucleon. It can be written as:

$$
J_{\mu}=\bar{v}\left(p_{1}\right)\left[G_{M}\left(q^{2}\right) \gamma_{\mu}+\frac{P_{\mu}}{M} F_{2}\left(q^{2}\right)\right] u\left(p_{2}\right),
$$

where $P_{\mu}=\left(p_{1}-p_{2}\right)_{\mu} / 2$, and $M$ is the hadron mass. The quantities $G_{M}\left(q^{2}\right)$ and $F_{2}\left(q^{2}\right)$ are the magnetic and Pauli FFs of the proton. They are complex functions of the variable $q^{2}$. The complex nature of FFs in the time-like region of momentum transfer is due to the strong interaction in the initial state. We use below the Sachs magnetic and charge FFs, which are related to the Dirac and Pauli FFs $F_{1,2}\left(q^{2}\right)$ as follows:

$$
G_{M}\left(q^{2}\right)=F_{1}\left(q^{2}\right)+F_{2}\left(q^{2}\right), G_{E}\left(q^{2}\right)=F_{1}\left(q^{2}\right)+\eta_{p} F_{2}\left(q^{2}\right), \eta_{p}=q^{2} /\left(4 M^{2}\right) .
$$

The differential cross section is related to the matrix element squared (3.1) by

$$
d \sigma=\frac{(2 \pi)^{4}}{4 \mathscr{I}} \overline{|\mathscr{M}|^{2}} \frac{d^{3} \vec{k}_{1} d^{3} \vec{k}_{2}}{(2 \pi)^{6} 4 E_{1} E_{2}} \delta^{4}\left(p_{1}+p_{2}-k_{1}-k_{2}\right),
$$

where $\mathscr{I}=\left(p_{1} \cdot p_{2}\right)^{2}-p_{1}^{2} p_{2}^{2}, E_{1}\left(E_{2}\right)$ is the energy of the $\ell^{-}\left(\ell^{+}\right)$lepton, and

$$
|\mathscr{M}|^{2}=\frac{e^{4}}{q^{4}} L_{\mu v} H_{\mu v}, L_{\mu \nu}=j_{\mu} j_{v}^{*}, H_{\mu v}=J_{\mu} J_{v}^{*}
$$


The expressions of the leptonic $L_{\mu \nu}$ and hadronic $H_{\mu \nu}$ tensors depend on the polarization states of the lepton and nucleon (spin $1 / 2$ particles). The density matrix $\rho=u(p) \bar{u}(p)$ for a free Dirac particle of four-momentum $p$ and mass $M$ can be written as:

$$
\rho=u(p) \bar{u}(p)=(\hat{p}+M) \frac{1}{2}\left(1-\gamma_{5} \hat{s}\right) .
$$

The polarization vector $s$ is a pseudo-vector which satisfies $s^{2}=-1$ and $s \cdot p=0$. It is related to the unit vector along polarization of the particle in its rest system, $\vec{\chi}$ by:

$$
s_{0}=\frac{\vec{p} \cdot \vec{\chi}}{M}, \vec{s}=\vec{\chi}+\frac{\vec{p}(\vec{p} \cdot \vec{\chi})}{M\left(M+p_{0}\right)} .
$$

\subsection{Electromagnetic tensors}

The leptonic tensor for the case of an unpolarized lepton is:

$$
L_{\mu \nu}^{(0)}=4\left(k_{1 \mu} k_{2 v}+k_{1 v} k_{2 \mu}\right)-2 q^{2} g_{\mu \nu},
$$

where $g_{\mu v}$ is the metric tensor of the Minkowski spacetime. The contribution to the electron tensor corresponding to a polarized electron is

$$
L_{\mu v}\left(s_{1}\right)=2 i m_{\ell} \varepsilon_{\mu v \rho \sigma} q_{\rho} S_{1 \sigma},
$$

where $S_{\sigma}$ is the polarization four-vector describing the lepton polarization, and $\varepsilon_{\mu v \rho \sigma}$ is the unit antisymmetric tensor with $\varepsilon_{0123}=1$.

The leptonic tensor can be written as a sum of unpolarized and polarized terms as:

$$
L_{\mu \nu}=L_{\mu \nu}^{(0)}+L_{\mu v}\left(s_{1}\right)+L_{\mu v}\left(s_{1}, s_{2}\right) .
$$

$L_{\mu v}\left(s_{1}, s_{2}\right)$ corresponds to the case when both leptons in the final state are polarized.

In the same way we can write the hadronic tensor:

$$
H_{\mu v}=H_{\mu v}^{(0)}+H_{\mu v}\left(s_{a}\right)+H_{\mu v}\left(s_{a}, s_{b}\right)
$$

The hadronic tensor for unpolarized protons, $H_{\mu \nu}^{(0)}$, is written as:

$$
H_{\mu v}^{(0)}=\left(g_{\mu v}-\frac{q_{\mu} q_{v}}{q^{2}}\right) H_{1}+P_{\mu} P_{v} H_{2}
$$

where

$$
H_{1}=-2 q^{2}\left|G_{M}\right|^{2}, H_{2}=\frac{8}{\eta_{p}-1}\left[\left|G_{M}\right|^{2}-\eta_{p}\left|G_{E}\right|^{2}\right] .
$$

The explicit expression of the tensor $H_{\mu v}\left(s_{a}\right)$ (antiproton beam is polarized) in terms of nucleon electromagnetic FFs is:

$$
\begin{aligned}
& H_{\mu \nu}^{(1)}\left(s_{a}\right)=-\frac{2 i}{M}\left[M^{2}\left|G_{M}\right|^{2}<\mu v q s_{a}>\right. \\
& \left.+\left(\eta_{p}-1\right)^{-1} \operatorname{Re} G_{M}\left(G_{E}-G_{M}\right)^{*}\left(<\mu p_{1} p_{2} s_{a}>P_{v}-<v p_{1} p_{2} s_{a}>P_{\mu}\right)\right] \\
& +\frac{2}{M\left(\eta_{p}-1\right)} \operatorname{Im} G_{M} G_{E}^{*}\left(<\mu p_{1} p_{2} s_{a}>P_{v}+<v p_{1} p_{2} s_{a}>P_{\mu}\right) .
\end{aligned}
$$


with the convention $\langle a b c d\rangle=\varepsilon_{\mu \nu \rho \sigma} a_{\mu} b_{v} c_{\rho} d_{\sigma}$ (the greek letters are used for summation indices, the roman letters indicate four-vectors).

The last term in Eq. $3.12 H_{\mu v}^{\left(s_{a}, s_{b}\right)}$ describes the annihilation of polarized antiproton beam with polarized proton target.

\section{Differential cross section and polarization observables}

The expression of the differential cross section for unpolarized $\bar{p} p \rightarrow \ell^{-} \ell^{+}$is obtained from the square of the scattering amplitude by the contraction of the unpolarized leptonic and hadronic tensors $\left(H_{\mu \nu}^{0} L_{\mu \nu}^{0}\right)$. The unpolarized cross section contains only the moduli squared of the form factors. The investigation of reaction (2.1) with polarized antiproton beam and/or polarized proton target carries information about the phase difference of the nucleon FFs, $\Phi=\Phi_{M}-\Phi_{E}$, where $\Phi_{M, E}=\arg G_{M, E}$.

The simplest polarization experiments is the polarization measurement of one of the scattered particles in unpolarized beam and target scattering, or the scattering of a polarized (unpolarized) beam on unpolarized (polarized) target without measurement of final state polarization. These experiments allow the measurement of single spin observables which are proportional to the imaginary part of nucleon FFs $\left(\operatorname{Im} F_{1} F_{2}^{*}\right)$.

\subsection{Single spin observable $A_{i}$}

Unlike elastic $e^{-} p$ scattering in one-photon exchange approximation, the hadronic tensor in the reaction (2.1) contains an antisymmetric part due to the fact that nucleon FFs are complex functions [6]. Therefore, in the present case, the polarization of only the antiproton may lead to nonzero spin asymmetry.

Single spin observables are obtained by the contraction of the spin-independent leptonic tensor $L_{\mu \nu}^{(0)}$ and the part of the hadronic tensor $L_{\mu \nu}^{(0)} H_{\mu \nu}^{(1)}\left(s_{1}\right)$, Eq. (3.15).

The single spin asymmetry due to the polarization of the lepton vanishes, because the spindependent leptonic tensor does not contain any symmetric part (over the indices $\mu$ and $v$ ). This is due to the fact that we assume that the electromagnetic interaction of the lepton is point-like (does not contain FFs). The measurement of this asymmetry constitutes an experimental test of the point-like nature of the $\tau\left(e\right.$ and $\mu$ )-lepton, at large values of $q^{2}$.

\subsection{Double spin polarization observables}

Three types of double spin observables are considered here:

- The polarization transfer coefficients $T_{i j}$, which describe the polarization transfer from the polarized antiproton beam to the produced negative lepton.

- Analyzing powers in the initial state $D_{i j}$ : polarized proton-antiproton collisions.

- Correlation coefficients in the final state $C_{i j}$ : polarized lepton-antilepton pair.

The indexes $i, j$ correspond to $n$ (normal), $t$ (transverse), $l$ (longitudinal), according to the direction of the polarization vectors of the lepton, or to $x, y, z$, referring to the direction of the 
hadron. The expressions for the double spin observables are combinations of $\operatorname{Im} F_{1} F_{2}^{*}, \operatorname{Re}_{1} F_{2}^{*}$ and $\left|F_{1,2}\right|^{2}$.

\subsection{Triple spin polarization observables}

The polarization observables for the production of a polarized (negative) lepton in the annihilation of polarized protons and antiprotons $M_{i 0 j k}$ are also calculated. The four subscripts denote, respectively, the detected particle, the associated particle, the projectile, and the target. These observables do not contain additional information about nucleon FFs, however the ratio of any pair of these triple spin polarization observables does not depend on the nucleon FFs, but is function only of kinematic variables. This is a consequence of the one-photon exchange approximation. In the case of the presence of two-photon exchange, this property does not hold anymore. Therefore, in principle, the measurement of these observables gives an indication of additional mechanisms beyond Born approximation.

\subsection{Polarized cross section}

The differential polarized cross section can be written in terms of the polarization observables as:

$$
\frac{d \sigma}{d \Omega}=\frac{d \sigma_{0}}{d \Omega}\left(1+A_{i} \chi_{1 i}+T_{i j} \chi_{1 i} \chi_{a j}+D_{i j} \chi_{1 i} \chi_{2 j}+C_{i j} \chi_{a i} \chi_{b j}+M_{i j k} \chi_{1 i} \chi_{2 j} \chi_{a k}\right),
$$

The factor $\frac{d \sigma_{0}}{d \Omega}$ is the unpolarized cross section. $\chi_{i, j, k}$ is the polarization vector along the $i, j$ or $k$ direction. The indices 1,2, $a$ and $b$ refer respectively to the antiproton, proton, negative and positive lepton.

The complete expressions of polarized and unpolarized cross section, taking into account the mass of the lepton, in CM and laboratory frame can be found in Ref. [11].

\section{Numerical Results}

The non-vanishing double spin observables are shown in Fig. 3, using the parametrization for the proton FFs of Ref. [12], as a function of $\cos \theta$ in CMS for $E=15 \mathrm{GeV}$.

In 1973 a model for the SL nucleon FFs was suggested by Iachello, Jackson and Lande [13] based on the Vector Meson Dominance, in which the external photon couples to the nucleon through intermediary vector mesons with the same quantum numbers as the photon (total spin 1 and odd parity $J^{P}=1^{-}$). In order to take into account the coupling to the vector mesons, the Dirac and Pauli nucleon FFs are expressed in terms of 4 isoscalar and isovector FFs. The proposed model is in excellent agreement with the polarization transfer data of the GEp collaboration [14]. In 2004, the generalisation was extended analytically to the TL region by introducing complex terms in the expressions of the FFs Ref. [12].

Fig. 3 shows that, for the $\tau$-lepton, the effect of the mass is sizable in all the observables. The difference between the $\mu$ and $e$ effects is tiny and it is best seen in the observables related to the transverse polarization of one lepton, such as $T_{t z}, T_{t z}, T_{n y}$, and $C_{l t}$. 

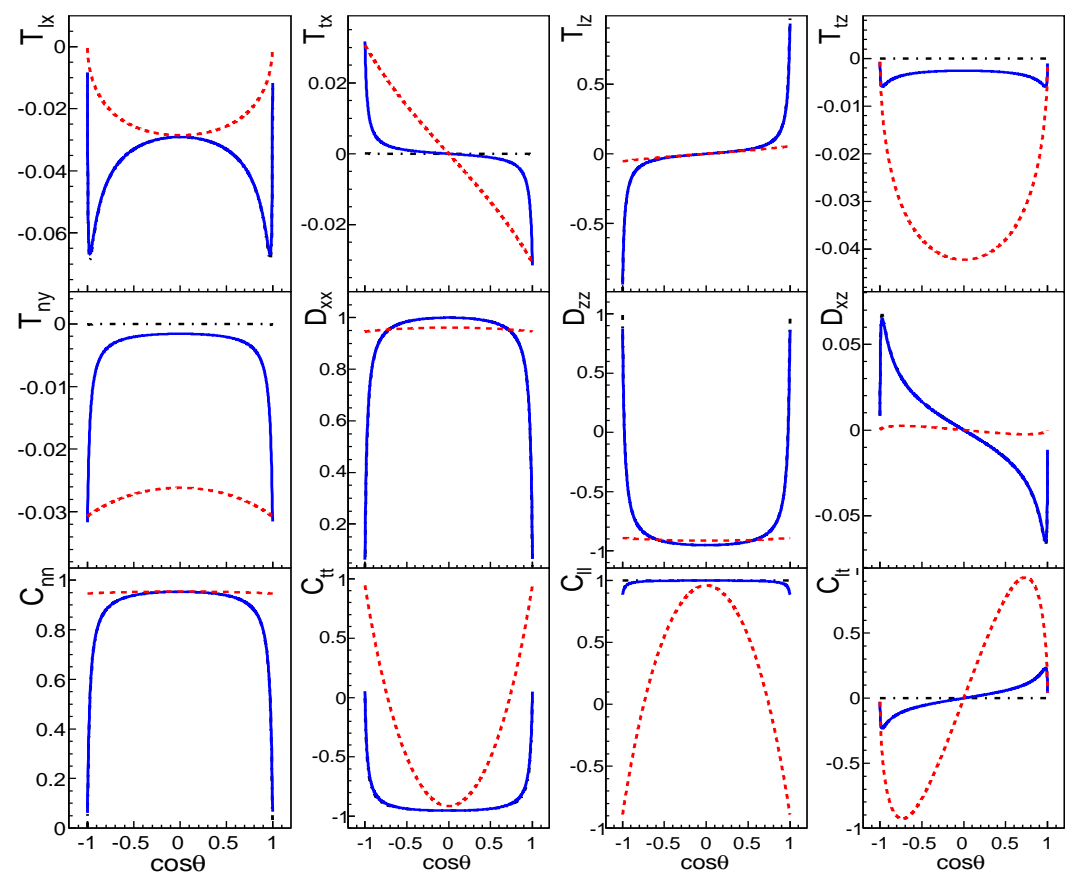

Figure 3: Double polarization observables as a function of $\cos \theta$, for $q^{2}=15 \mathrm{GeV}^{2}$, using the parametrization from Ref. [12] in CMS, for $\mu$ (blue solid line), for $e$ (black dash-dotted line), and for $\tau$ (red dotted line). The calculation for $e$ is hardly visible in some observables since it overlaps with the $\mu$ line.

The coefficients $T_{n y}^{C}, T_{t x}^{C}, T_{t z}^{C}$ are proportional to the mass of the produced lepton and they are suppressed by the factor $m_{\ell} / M$ for $\ell=e$ or $\mu$. In the case of a $\tau$-lepton this factor constitutes an enhancement of $\sim 2$.

\section{Conclusion}

The measurement of TL proton FFs at PANDA will enhance our knowledge on the electromagnetic structure of proton. The heavy lepton production allows a precise measurement of proton FFs by reducing the effect of photon radiation. Heavy lepton production enhances the polarization observables of transverse polarized lepton. The measurement of these observables gives access to the relative phase of FFs. The measurements of these polarization observables and the proton TL FFs at PANDA using the production of heavy leptons is under investigation.

\section{References}

[1] Physics Performance Report for PANDA: Strong Interaction Studies with Antiprotons, The PANDA Collaboration, arXiv:0903.3905 [hep-ex]; http://www.gsi.de/PANDA;

[2] Facility for Antiproton and Ion Research, http://www.fair-center.eu.

[3] M. Sudol, M. C. Mora Espi, E. Becheva, J. Boucher, T. Hennino, R. Kunne, D. Marchand and S. Ong et al., Eur. Phys. J. A 44, 373 (2010). 
[4] A. Zichichi, S. M. Berman, N. Cabibbo, R Gatto, Nuovo Cim. 24 (1962) 170.

[5] S. M. Bilenkii, C. Giunti and V. Wataghin, Z. Phys. C59 (1993) 475.

[6] G. I. Gakh, E. Tomasi-Gustafsson, Nucl. Phys. A761 (2005) 120.

[7] A. I. Ahmadov, V. V. Bytev, E. A. Kuraev and E. Tomasi-Gustafsson, Phys. Rev. D 82 (2010) 094016.

[8] http://www-panda.gsi.de/db/notesDBr/AD44-121211_RCsim.pdf

[9] D. Decamp et al. [ ALEPH Collaboration ], Phys. Lett. B265 (1991) 430.

[10] A. Heister et al. [ ALEPH Collaboration ], Eur. Phys. J. C20 (2001) 401.

[11] A. Dbeyssi, E. Tomasi-Gustafsson, G. I. Gakh and M. Konchatnyi, Nucl. Phys. A 894 (2012) 20.

[12] F. Iachello and Q. Wan, Phys. Rev. C 69 (2004) 055204.

[13] F. Iachello, A. D. Jackson and A. Lande, Phys. Lett. B 43 (1973) 191.

[14] A. J. R. Puckett et al., Phys. Rev. Lett. 104 (2010) 242301 and Refs therein. 